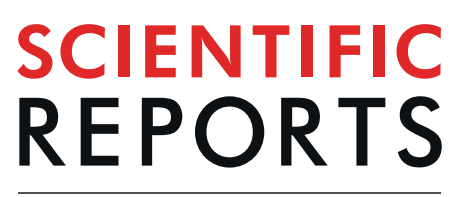

natureresearch

There are amendments to this paper

\title{
Systemic inflammation is associated with incident stroke and heart disease in East Asians
}

Mohd A. Karim ${ }^{1}{ }^{1}$, Christiana Kartsonaki ${ }^{1,2}$, Derrick A. Bennett ${ }^{1}$, lona Y. Millwood ${ }^{1,2}$, Michael R. Hill ${ }^{1,2}$, Daniel Avery ${ }^{1}$, Zheng Bian ${ }^{3}$, Huaidong Du ${ }^{1,2}$, Yu Guo $^{3}$, Yijian Oian ${ }^{4}$, Chan $\mathrm{Qu}^{5}$, lain Turnbull ${ }^{1}{ }^{1}$, Dan Schmidt-Valle ${ }^{1}$, Chunmei Wang ${ }^{4}$, Canqing Yu ${ }^{3}$, Jun Lv ${ }^{6}$, Junshi Chen ${ }^{7}$, Robert Clarke ${ }^{1}$, Liming Li ${ }^{6}$, Zhengming Chen $\mathbb{1}^{1}{ }^{1}$, Michael V. Holmes ${ }^{1,2,8,9 *}$, Robin G. Walters $\mathbb{1}^{1,2,9 *}$ \& China Kadoorie Biobank Collaborative Group ${ }^{+}$

Systemic inflammation, reflected by increased plasma concentrations of C-reactive protein (CRP) and fibrinogen, is associated with increased risk of coronary heart disease, but its relevance for stroke types remains unclear. Moreover, evidence is limited in non-European populations. We investigated associations of CRP and fibrinogen with risks of incident major coronary events (MCE), ischemic stroke (IS) and intracerebral hemorrhage $(\mathrm{ICH})$ in a cohort of Chinese adults. A nested case-control study within the prospective China Kadoorie Biobank included 1,508 incident MCE cases, 5,418 IS cases, 4,476 ICH cases, and 5,285 common controls, aged 30-79 years. High-sensitivity CRP and low-density lipoprotein cholesterol (LDL-C) were measured in baseline plasma samples from all participants, and fibrinogen in a subset $(n=9,380)$. Logistic regression yielded adjusted odds ratios (ORs) per SD higher usual levels of log-transformed CRP and fibrinogen. The overall mean (SD) baseline LDL-C was $91.6 \mathrm{mg} / \mathrm{dL}$ (24.0) and geometric mean $(95 \% \mathrm{Cl}) \mathrm{CRP}$ and fibrinogen were $0.90 \mathrm{mg} / \mathrm{L}(0.87-0.93)$ and 3.01 g/L (2.98-3.03), respectively. There were approximately log-linear positive associations of CRP with each outcome, which persisted after adjustment for LDL-C and other risk factors, with adjusted ORs $(95 \% \mathrm{CI})$ per SD higher CRP of 1.67 (1.44-1.94) for MCE and 1.22 (1.10-1.36) for both IS and ICH. No associations of fibrinogen with MCE, IS, or ICH were identified. Adding CRP to prediction models based on established risk factors improved model fit for each of $M C E$, IS, and ICH, with small improvements in C-statistic and correct reclassification of controls to lower risk groups. Among Chinese adults, who have low mean LDLC, CRP, but not fibrinogen, was independently associated with increased risks of MCE and stroke.

Coronary heart disease (CHD) and stroke are the leading causes of premature death globally ${ }^{1}$. In addition to traditional risk factors, such as smoking, blood pressure and $\mathrm{LDL}-\mathrm{C}^{2}$, there is accumulating evidence that inflammation plays an important role in the etiology of cardiovascular disease (CVD) $)^{3}$.

The local inflammatory response following injury to the arterial endothelium is regarded as a key step in the initiation of atherosclerotic $\mathrm{CVD}^{4}$. Injury triggers a cascade that recruits immune cells (monocytes and macrophages), promotes oxidation and aggregation of low-density lipoprotein cholesterol (LDL-C) and contributes to development of atherosclerotic plaques ${ }^{5,6}$. The process may be amplified by elevated levels of circulating pro-inflammatory cytokines ${ }^{7}$ and, hence, persistent low-grade systemic inflammation has emerged as an important modifiable risk factor for atherosclerosis ${ }^{8,9}$.

${ }^{1}$ Clinical Trial Service Unit \& Epidemiological Studies Unit (CTSU), Nuffield Department of Population Health, University of Oxford, Oxford, United Kingdom. ${ }^{2}$ Medical Research Council Population Health Research Unit (MRC PHRU) at the University of Oxford, Nuffield Department of Population Health, University of Oxford, Oxford, United Kingdom. ${ }^{3}$ Chinese Academy of Medical Sciences, Beijing, China. ${ }^{4}$ Tongxiang Centre for Disease Control and Prevention, Tongxiang, Zhejiang Province, China. ${ }^{5}$ NCDs Prevention and Control Department, Liuyang Centre for Disease Control and Prevention, Liuyang, Hunan Province, China. ${ }^{6}$ Department of Epidemiology, School of Public Health, Peking University Health Science Center, Beijing, China. ${ }^{7}$ China National Center for Food Safety Risk Assessment, Beijing, China. ${ }^{8}$ National Institute for Health Research Oxford Biomedical Research Centre, Oxford University Hospital, Oxford, United Kingdom. ${ }^{9}$ Michael V. Holmes and Robin G. Walters are listed as jointly supervising this work. ${ }^{\dagger} A$ comprehensive list of consortium members appears at the end of the paper. *email: michael.holmes@ndph.ox.ac.uk; robin.walters@ndph.ox.ac.uk 
Circulating C-reactive protein (CRP) and fibrinogen, regarded as markers of systemic inflammation, are associated with increased risk of atherosclerosis ${ }^{10-16}$ and atherosclerotic CVDs including CHD and ischemic stroke (IS) ${ }^{17-21}$. Most of the available evidence has been derived from observational studies of Western populations in whom LDL-C levels are high (mean $\sim 140 \mathrm{mg} / \mathrm{dL})^{17}$. While Mendelian randomization (MR) studies have indicated that CRP and fibrinogen are unlikely to be causal in atherosclerotic CVD ${ }^{22-26}$, such inflammatory markers are nevertheless useful indicators of the activities of pro-inflammatory cytokines that promote their synthesis. For example, the Canakinumab Anti-inflammatory Thrombosis Outcome Study (CANTOS) trial, in which inhibition of the pro-inflammatory cytokine interleukin-1 beta (IL-1 $\beta$ ) provided the first evidence from a randomized controlled trial (RCT) for a role of inflammation in $\mathrm{CVD}^{9}$, assessed IL- $1 \beta$ activity via measurements of CRP and fibrinogen levels ${ }^{27}$.

Compared to the well-established associations of CRP with risk of CHD, observational evidence concerning the relevance of inflammation to individual stroke types is limited. In particular, there is limited evidence on the associations of systemic inflammation, including CRP and fibrinogen, with risk of intracerebral hemorrhage (ICH). Reliable assessment of the association of systemic inflammation with stroke types is particularly important in China, where stroke rates are much higher than for Western populations, particularly for $\mathrm{ICH}^{28}$, in spite of relatively low mean LDL-C levels $(\sim 92 \mathrm{mg} / \mathrm{dL})$. To address these questions, we examined the associations of CRP and fibrinogen with risks of incident major coronary events (MCE), IS, and ICH in a nested case-control study, set within the prospective China Kadoorie Biobank (CKB).

\section{Results}

Baseline characteristics of study participants. Table 1 shows the characteristics of the study participants. Controls (mean age 55.8 years, $49.8 \%$ female) were older and had a higher proportion of men than the overall CKB population $^{29}$, reflecting the study design for the nested case-control cohort from which the study population was drawn. Cases of IS (55.4 years) and MCE (56.5 years) were similar in age to controls but ICH cases (59.3 years) were older. Although the proportion of women was similar between controls (49.6\%) and ICH cases (49.9\%), a lower proportion of women had MCE (41.6\%) whilst a higher proportion had an IS event (55.6\%). Systolic blood pressure (mmHg) was higher in MCE (143.6) and IS (144.4) cases than in controls (134.4), but was highest in ICH cases (152.9). Compared to controls, larger proportions of MCE and stroke cases reported poor health (MCE: 18\%; IS: 15\%; ICH: 16\%; controls: 8\%), hypertension (MCE: 22\%; IS: 24\%; ICH: 26\%; controls: $10 \%$ ), and diabetes (MCE: 14\%; IS: 12\%; ICH: 9\%; controls: 6\%). There were, however, no appreciable differences in adiposity traits (BMI, waist-hip ratio) or levels of self-reported physical activity between cases and controls.

Markers of inflammation (CRP and fibrinogen), lipids (LDL-C and TG), and subclinical atherosclerosis (proportion of participants with plaque, carotid intima media thickness) were higher in participants with MCE and IS (i.e. atherosclerotic CVD) than in controls. By contrast, differences in these traits were smaller (for CRP and plaque) or absent between ICH cases and controls.

Cross-sectional associations of CRP and fibrinogen. Both CRP and fibrinogen showed strong associations with a range of key CVD risk factors and potential confounders. In control individuals there were positive associations of CRP with LDL-C $(P$-trend $=0.016)$, TG $\left(P\right.$-trend $\left.=5.3 \times 10^{-3}\right)$, and BMI $\left(P\right.$-trend $\left.=9.3 \times 10^{-11}\right)$, a less strong positive association with SBP $(P$-trend $=0.06)$, and inverse dose-response associations with HDL-C $\left(P\right.$-trend $\left.=1.8 \times 10^{-5}\right)$ and physical activity $(P$-trend $=0.028)($ Figs. $1, \mathrm{~S} 2) . \mathrm{CRP}$ also showed positive cross-sectional associations with markers of liver (AST, ALT, and GGT) and renal function (creatinine, uric acid, and cystatin-C), and ApoB, and Lp(a), inverse associations with HDL-C, ApoA1 and albumin, and no association with total vitamin D (Fig. S2). A similar pattern of associations was seen for fibrinogen, except that no association was observed for TG. Further cross-sectional associations of CRP and fibrinogen were observed with a wide range of population baseline characteristics, in particular strong positive associations with age, with differences between the top and bottom age categories of $0.50 \mathrm{mg} / \mathrm{L}\left(P=1.16 \times 10^{-126}\right)$ for CRP and $0.61 \mathrm{~g} / \mathrm{L}\left(P=5.42 \times 10^{-307}\right)$ for fibrinogen, representing the impact of a mean difference of 24 years (Table S2).

Associations of CRP and Fibrinogen with MCE and Stroke types. There were log-linear positive associations of $\log$ CRP with risks of MCE, IS and ICH (Fig. 2), with adjusted ORs [95\% CI] per-SD higher usual $\log$ CRP of 2.05 [1.80-2.33] for MCE, 1.57 [1.43-1.73] for IS, and 1.43 [1.30-1.56]) for ICH in the base model (adjusted only for age, age ${ }^{2}$ and sex) (Fig. 3). For each, the association was attenuated after adjustment for SBP, but the association persisted even after further adjustment for BMI, lipids (LDL-C and TG) and other CVD risk factors (Figs. 3, S4(A)). After adjusting for LDL-C the association of CRP with MCE was attenuated to 1.77 [1.54-2.04], and in the fully adjusted model was 1.67 [1.44-1.94]. Similarly, the associations of IS and ICH with CRP after full adjustment were attenuated to 1.22 [1.10-1.36] and (again) 1.22 [1.10-1.36], respectively.

We further assessed whether associations of log CRP with MCE, IS and ICH risk differed within pre-specified subgroups of environmental, behavioral, or physiological factors. There was no evidence (after Bonferroni adjustment, $P<0.002$ ) for effect modification by any of these factors, including by LDL-C (Fig. 4).

By contrast, although fibrinogen levels were associated with MCE and IS in the base model, the association attenuated to the null after further adjustment (MCE, OR: 1.10 [0.90-1.36]; IS, OR: 1.00 [0.84-1.19], per-SD higher usual log fibrinogen); with ICH, no association was observed, even in the base model (Figs. 2, S3,S4). Associations of fibrinogen with MCE, IS, and ICH risk did not exhibit differences between most of the pre-specified subgroups, including of lipids; however, there was evidence of heterogeneity by levels of physical activity $\left(P=6.7 \times 10^{-4}\right)$ for MCE and suggestive evidence of an interaction with smoking $(P=0.0021)$ for IS (Fig. S5). 


\begin{tabular}{|c|c|c|c|c|c|}
\hline Trait & $\begin{array}{l}\text { Controls } \\
(\mathrm{n}=5285)\end{array}$ & $\begin{array}{l}\text { Major Coronary } \\
\text { Events }(n=1508)\end{array}$ & $\begin{array}{l}\text { Ischemic Stroke } \\
(\mathrm{n}=5418)\end{array}$ & \multicolumn{2}{|c|}{$\begin{array}{l}\text { Intracerebral Hemorrhage } \\
(\mathrm{n}=4476)\end{array}$} \\
\hline \multicolumn{6}{|c|}{ Age and Sociodemographic factors } \\
\hline Age, $y^{*}$ & $55.8(10.9)$ & $56.5(9.72)$ & $55.4(9.36)$ & \multicolumn{2}{|c|}{$59.3(10.1)$} \\
\hline Female $^{\dagger}, \%$ & 49.6 & 41.6 & 55.6 & \multicolumn{2}{|c|}{49.9} \\
\hline Rural $^{\ddagger}, \%$ & 79.2 & 66.6 & 54.6 & \multicolumn{2}{|c|}{79.5} \\
\hline Middle School and above, $\%$ & 38.3 & 36.8 & 38.2 & \multicolumn{2}{|c|}{36.0} \\
\hline Income $>10,000$ yuan/year, $\%$ & 62.2 & 61.5 & 65.6 & \multicolumn{2}{|c|}{62.5} \\
\hline \multicolumn{6}{|l|}{ Lifestyle factors } \\
\hline Male smokers, $\%$ & 87.1 & 90.9 & 89.5 & \multicolumn{2}{|c|}{86.9} \\
\hline Female smokers, $\%$ & 3.9 & 7.8 & 5.4 & \multicolumn{2}{|c|}{5.9} \\
\hline Male regular drinkers, $\%$ & 32.1 & 26.5 & 30.9 & \multicolumn{2}{|c|}{33.1} \\
\hline Female regular drinkers, $\%$ & 2.1 & 1.8 & 2.2 & 2. & \\
\hline Physical activity, MET h/day & $20.1(11.8)$ & $18.7(11.6)$ & $18.7(11.5)$ & & $(11.6)$ \\
\hline Anthropometric, blood pressu & circulatory measur & & & & \\
\hline Standing height, $\mathrm{m}$ & $1.59(0.06)$ & $1.59(0.06)$ & $1.59(0.06)$ & & $(0.06)$ \\
\hline BMI, kg/m² & $23.4(3.1)$ & $23.9(3.4)$ & $24.1(3.4)$ & & $(3.4)$ \\
\hline Waist-hip ratio & $0.89(0.07)$ & $0.91(0.07)$ & $0.90(0.07)$ & & $(0.07)$ \\
\hline SBP, mmHg & $134.4(19.7)$ & $143.6(25.1)$ & $144.4(23.9)$ & & $(27.3)$ \\
\hline DBP, mmHg & $78.5(11.0)$ & $83.0(13.3)$ & $83.9(12.5)$ & & $(14.2)$ \\
\hline Heart rate, per minute & $78.3(11.7)$ & $80.4(12.7)$ & $79.2(12.1)$ & & $(12.6)$ \\
\hline Markers of subclinical atheros & & & & & \\
\hline Plaque, $\%$ & 39.9 & 60.5 & 50.7 & 44 & \\
\hline cIMT, mm & $0.71(0.13)$ & $0.78(0.23)$ & $0.75(0.16)$ & & $(0.16)$ \\
\hline Inflammatory biomarkers & & & & & \\
\hline $\mathrm{CRP}, \mathrm{mg} / \mathrm{L}^{\S}$ & $0.90(0.86-0.93)$ & $1.31(1.24-1.39)$ & $1.12(1.08-1.16)$ & & $(1.04-1.13)$ \\
\hline Fibrinogen, $\mathrm{g} / \mathrm{L}^{\mathrm{S}}$ & $3.00(2.98-3.03)$ & $3.07(3.04-3.11)$ & $3.05(3.02-3.08)$ & & $(2.99-3.05)$ \\
\hline Lipids & & & & & \\
\hline Triglycerides $\$$, mg/dL & $145.8(143.1-148.5)$ & $163.4(158.5-168$ & \begin{tabular}{l|l}
$5)$ & $156.6(153.9-1$ \\
\end{tabular} & $9.45)$ & $145.5(142.7-148.5)$ \\
\hline LDL cholesterol?, mg/dL & $91.6(23.9)$ & $95.9(29.1)$ & $95.9(25.8)$ & & $90.8(26.4)$ \\
\hline Self-reported health and disea & & & & & \\
\hline Poor self-rated health, $\%$ & 8.1 & 18.4 & 14.6 & 15 & \\
\hline Hypertension, \% & 10.4 & 21.7 & 23.7 & 26 & \\
\hline Diabetes, $\%$ & 6.0 & 14.0 & 12.4 & 9. & \\
\hline
\end{tabular}

Table 1. Baseline traits of study participants. CRP: C-reactive protein; cIMT: carotid intima-media thickness; DBP: Diastolic Blood Pressure; ICH: Intracerebral hemorrhage; IS: Ischemic stroke; LDL: Low-density lipoprotein; MET: metabolic equivalents; SBP: Systolic Blood Pressure. Values are mean (standard deviation) for continuous traits or proportion (weighted percentage) for binary/categorical traits, standardized to age, sex and study area of case-control cohort unless otherwise stated. *Standardized to sex and study area only. ${ }^{\dagger}$ Standardized to age and study area only. ${ }^{\ddagger}$ Standardized to age and sex only. ${ }^{\circledR}$ Geometric mean (95\% CI). ${ }^{~}$ Note: Low-density lipoprotein (LDL) cholesterol was directly measured.

Discriminatory performance. Addition of CRP to a logistic regression model, comprising age, sex, region, smoking, diabetes, BMI, SBP, LDL-C, HDL-C, and TG, yielded small improvements in the C-statistic for MCE (0.7181[95\% CI: $0.7028-0.7334$ ] to 0.7251 [95\% CI: 0.7101-0.7402]) which were significant by DeLong's test, but there were smaller differences in the AUCs for IS and ICH (Table S3). Nevertheless, a likelihood ratio test (LRT) - which is a more powerful test of model fit than the rank-based $\mathrm{C}$ statistic ${ }^{30}$ - identified improved model fit for MCE $\left(\right.$ LRT $\left.p=1.21 \times 10^{-13}\right)$, IS $\left(\right.$ LRT $\left.p=1.75 \times 10^{-4}\right)$ and ICH $\left(p=9.54 \times 10^{-7}\right)$. Marginal net reclassification improvements (NRI) were observed for MCE (NRI $=0.035$ [95\% CI: $0.014,0.056], p=0.0011])$, IS (NRI $=0.011$ [95\% CI: $0.054,0.016], \mathrm{p}=8.0 \times 10^{-5}$ ]), and ICH $(\mathrm{NRI}=0.0064$ [95\% CI: 0.0011, 0.0117], $\left.\mathrm{p}=0.019]\right)($ Table S4), showing that in each case the model improvement predominantly reflected improved reclassification of controls to lower risk; a similar improvement in the reclassification of cases was not seen.

\section{Discussion}

In this large nested case-control study of Chinese adults, who have low mean LDL-C ( 92 mg/dL), CRP was positively associated with risks of incident MCE, IS, and $\mathrm{ICH}$, independent of established risk factors. The magnitude of the increase in risk was greater for MCE than for stroke, was similar for IS and ICH, and was consistent across different population subgroups. By contrast, after adjustment for other risk factors we observed no increase in risk associated with higher levels of fibrinogen. The findings of this large study extend the well-established association in Europeans of CRP with atherosclerotic disease, and further support a role for systemic inflammation in vascular disease, independent of lipids. 

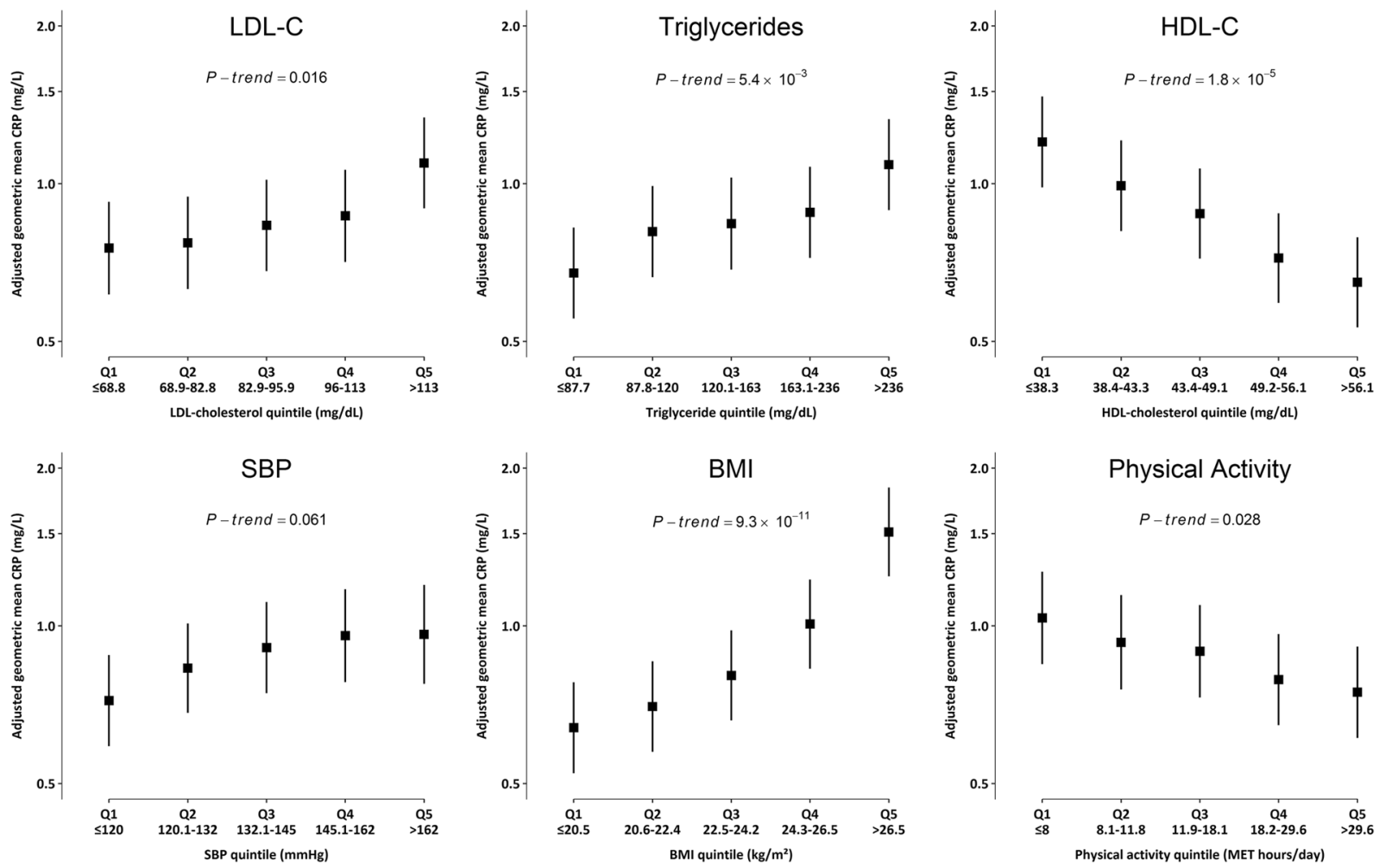

Figure 1. Cross-sectional associations of high-sensitivity C-reactive protein (CRP) with selected cardiovascular disease (CVD) risk factors. Analyses were carried out only in controls $(n=5,285)$. Cut-offs for biomarker quintiles were based on their distribution in controls only. Geometric mean of CRP was adjusted for age, age ${ }^{2}$, sex and region. $P$-trend was calculated using chi-square test statistic with 1 degree of freedom. Error bars represent $95 \%$ CI for each quintile.

Our findings for CRP are broadly consistent with previous prospective studies conducted in both European and East Asian populations which have consistently demonstrated strong positive associations of CRP with risk of CHD and ischemic stroke ${ }^{17,19-21}$, but with at best marginal improvements in risk prediction ${ }^{31,32}$. In a large meta-analysis of 54 prospective studies in populations of European ancestry, involving 5,373 CHD cases (first-ever MI or fatal CHD) and 1,931 IS cases, each 1 SD higher usual log CRP concentration was associated with $37 \%$ and $27 \%$ higher risk of CHD and IS, respectively. Our corresponding estimate for IS was very similar (22\%), but our MCE estimate (67\%) was appreciably greater than in the meta-analysis, with very little overlap of confidence intervals. This perhaps reflects a different spectrum of disease severity and/or aetiology - there were a much higher proportion of fatal MCE cases in the present study (65\% vs $29 \%$ ).

An important rationale for our study was to investigate whether inflammatory markers have different relationships with the pathophysiologically distinct IS and ICH stroke types ${ }^{33}$. Previous studies have highlighted their differing, even opposing, relationships with some CVD risk factors such as LDL-C ${ }^{34}$ or BMI ${ }^{35}$. By contrast, we found associations of CRP with IS and ICH that were concordant in both direction and strength. These results differ from previous studies which reported null associations of CRP with ICH, although these included relatively few ICH cases ( 383 to 738 in previous studies vs 4,476 in our study), typically leading to effect estimates with wide confidence intervals that overlap our estimate of a $27 \%$ increase in risk per SD higher usual log CRP concentration $^{19-21}$. In separate analyses in CKB, other markers of inflammation that are only moderately correlated with CRP, such as glycoprotein acetyls ${ }^{36}$, also exhibited positive associations with both IS and ICH, providing additional support for the role of inflammation in both stroke types ${ }^{37}$.

Unexpectedly, for fibrinogen, associations with both MCE and IS when adjusting only for age and sex were attenuated to the null after further adjustment for a range of potential confounders. A large meta-analysis of 31 prospective studies, involving a total of 154,211 participants (including 7\% from Japan), demonstrated positive associations with CHD and overall stroke (mainly IS) which, although partially attenuated, remained strong after adjustment for classical risk factors, including smoking, alcohol, BMI, LDL-C, TG, and SBP, although there remained the possibility of substantial residual confounding ${ }^{18}$. Such residual confounding could potential account for the apparent discrepancy between the two studies. Alternatively, the apparent disparity between the results for CRP and fibrinogen perhaps reflects that they reflect non-identical components of systemic inflammation. This is supported by the strong association in our study between TG and CRP, yet there was no such relationship between TG and fibrinogen. Importantly, the previous meta-analysis that reported an association of fibrinogen with CVD also showed a strong positive association of fibrinogen with $\mathrm{TG}^{18}$.

These findings have potential implications for treatment strategies to modify inflammation for prevention of vascular disease $\mathrm{s}^{9,38,39}$. Importantly, the CANTOS trial of $\sim 10,000$ patients with prior MI ( $>90 \%$ on statins, median 
CRP

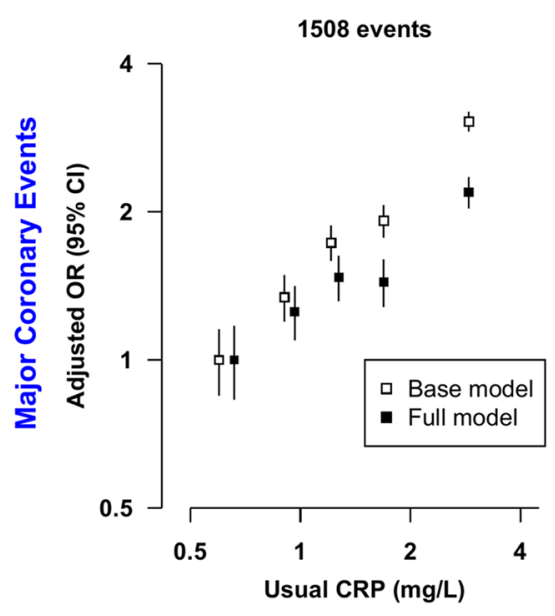

5418 events
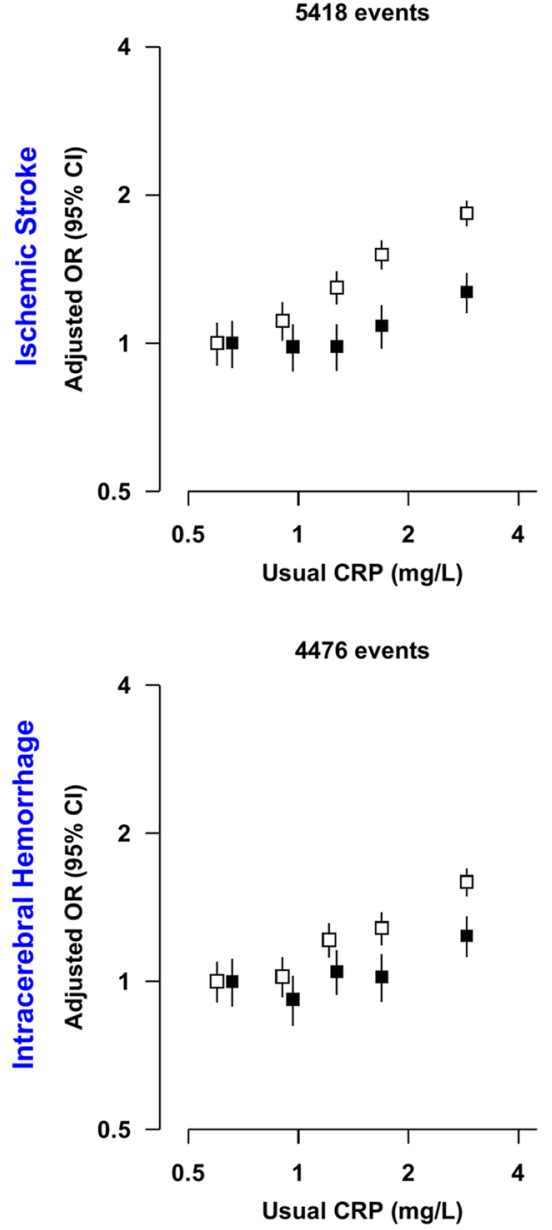

Fibrinogen

1250 events

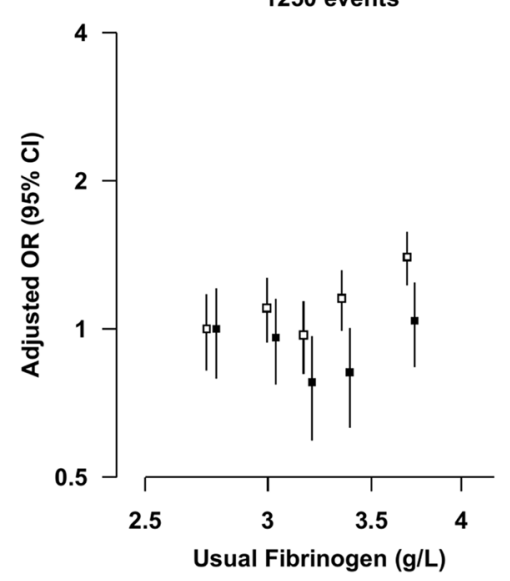

2280 events

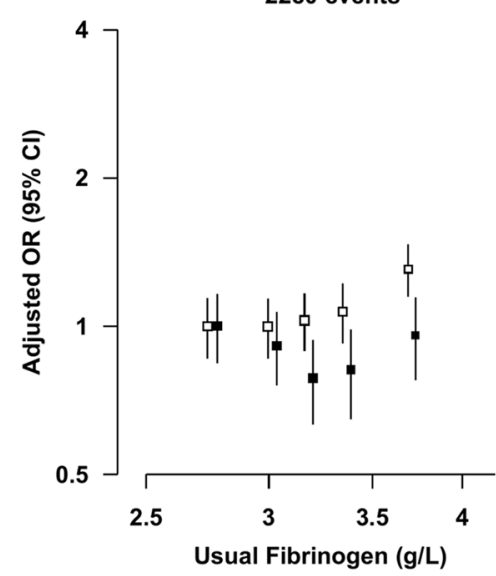

1998 events

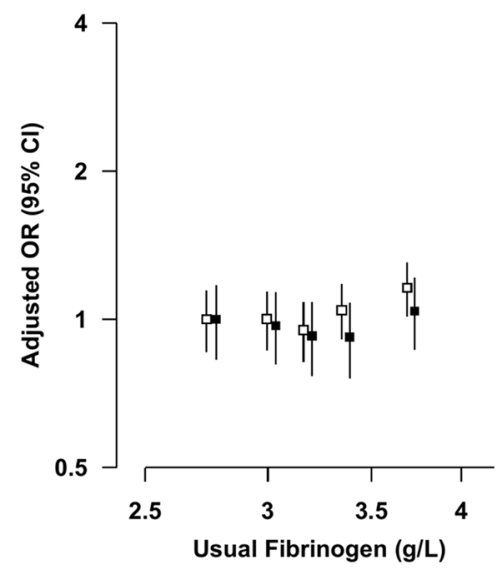

Figure 2. Risk of Major Coronary Events, Ischemic Stroke, and Intracerebral hemorrhage by quintiles of C-reactive protein (CRP) and fibrinogen. Models were adjusted for age, age ${ }^{2}$ and sex (Base model) and additionally for income, occupation, education, SBP, BMI, diabetes, physical activity, standing height, smoking, alcohol, LDL-C and TG (Full model). These models were fitted separately for each of the 10 study areas for each quintile and estimates were meta-analyzed; in each quintile, study areas were excluded when models failed to converge ( 9 models out of 600 models). Cut-offs for CRP and Fibrinogen quintiles were based on their distribution in the combined case-control cohort (CRP: $0.37 \mathrm{mg} / \mathrm{l}, 0.70 \mathrm{mg} / \mathrm{l}, 1.28 \mathrm{mg} / \mathrm{l}$, and $2.59 \mathrm{mg} / \mathrm{l}$; Fibrinogen: $2.47 \mathrm{~g} / \mathrm{l}, 2.81 \mathrm{~g} / \mathrm{l}, 3.14 \mathrm{~g} / \mathrm{l}$, and $3.60 \mathrm{~g} / \mathrm{l})$. Error bars represent $95 \%$ floated confidence intervals (CI). The areas of the boxes are proportional to the inverse of the variance of the log ORs. OR, CRP, and fibrinogen are plotted on log scales. 
(A) Major Coronary Events

\section{Model}

Base model (Model 1)

Model $1+$ BMI and SBP

Model $1+$ Lipids (LDL-C and TG)

Model $1+$ Other CVD risk factors

Full model

(B) Ischemic Stroke

\section{Model}

Base model (Model 1)

Model $1+$ BMl and SBP

Model $1+$ Lipids (LDL-C and TG)

Model $1+$ Other CVD risk factors

Full model

(C) Intracerebral hemorrhage

\section{Model}

Base model (Model 1)

Model $1+$ BMl and SBP

Model $1+$ Lipids (LDL-C and TG)

Model $1+$ Other CVD risk factors

Full model

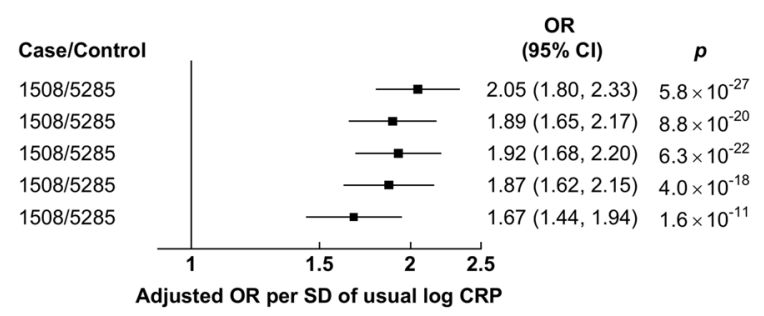

Adjusted OR per SD of usual log CRP

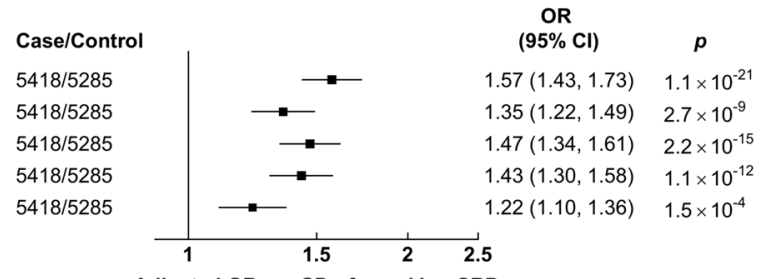

Adjusted OR per SD of usual log CRP

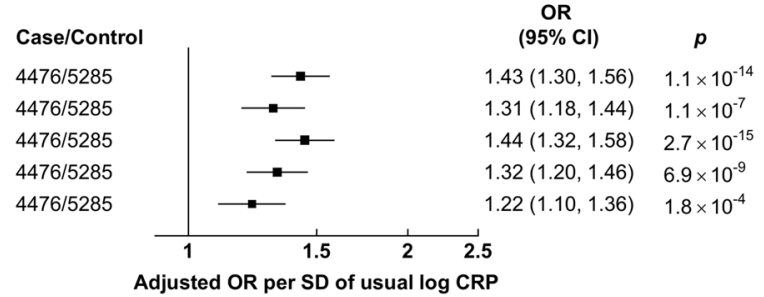

Figure 3. Risk of (A) Major Coronary Events, (B) Ischemic Stroke, and (C) Intracerebral hemorrhage per standard deviation (SD) of usual log-transformed high-sensitivity C-reactive protein (CRP). Models were adjusted for age, age ${ }^{2}$ and sex (base model) and additionally for income, occupation, education, SBP, BMI, diabetes, physical activity, standing height, smoking, alcohol, LDL-C and TG (Full model). The models were fitted separately for each of the 10 study areas and estimates were meta-analyzed. Error bars represent $95 \%$ confidence intervals (CI). The areas of the boxes are proportional to the inverse of the variance of the log ORs.

baseline LDL-C levels in placebo group: $83 \mathrm{mg} / \mathrm{dL}$ ) demonstrated a beneficial effect of therapeutic inhibition of IL-1 $\beta$ on the primary endpoint of major vascular events. Compared with placebo, allocation to canakinumab was associated with pooled hazard ratios $(95 \% \mathrm{CI})$ for $\mathrm{MI}$ and stroke of 0.84 [0.73-0.97] and 0.93 [0.72-1.20], respectively. While treatment with canakinumab did not lead to a reduction in risk of stroke, this may reflect the lack of power rather than representing a true null effect, since only 264 stroke cases accrued during the 48 month trial duration. Thus, although individuals in the present observational study were drawn from the general population (as opposed to being a study of patients with a previous MI as in the CANTOS interventional trial), were not on statins, and had with a median baseline LDL-C levels (in controls) of $86 \mathrm{mg} / \mathrm{dL}$, our data confirm the potential importance of inflammation pathways for stroke and MCE, independent of LDL-C.

A key strength of our study lies in the large number of well-characterized stroke cases. The majority $(90 \%)$ had CT or MRI scans at the time of hospital admission ${ }^{40}$; further adjudication by clinician-led review of medical records refuted the diagnosis in only $7 \%$ of cases with IS and $8 \%$ with $\mathrm{ICH}$. In addition, we measured CRP and fibrinogen using plasma samples of study participants collected at baseline, prior to the occurrence of incident vascular events, thus somewhat limiting reverse causality bias. Further, we were able to correct for measurement error and within-person variation in CRP and fibrinogen levels (i.e. regression dilution bias) by using measurements from repeat plasma samples collected 6-9 years later, so that our results reflect the risk associated with usual levels of these biomarkers.

Study limitations. The associations of CRP with risk of incident MCE, IS and ICH were attenuated by inclusion of additional risk factors, and it is possible that there remains residual confounding due to inaccurate measures of several such potential confounders (e.g. physical activity), or biases from other sources such as reverse causality. However, the impact of such confounding seems likely to be limited, and the prospective study design likely limits reverse causation. Conversely, the lack of association of fibrinogen with MCE and stroke types in our study may reflect limited statistical power. While, the present study included twice the number of IS cases compared to the largest fibrinogen meta-analysis to date (our study vs meta-analysis, 2,280 vs 1,370 IS cases in fully adjusted models) ${ }^{18}$, we are currently unable to determine the contributions of different stroke sub-types. Associations of CRP and fibrinogen may not be consistent across subtypes of IS (e.g. large artery, small vessel, and cardio-embolic) and ICH (e.g. lobar and non-lobar), something that will be investigated once ongoing characterization of stroke subtypes in CKB is complete.

It should be emphasized that CRP does not play a causal role in stroke, as indicated by large-scale evidence from Mendelian randomization studies ${ }^{22,24}$. Rather, the consistent observed associations of CRP with MCE, IS 


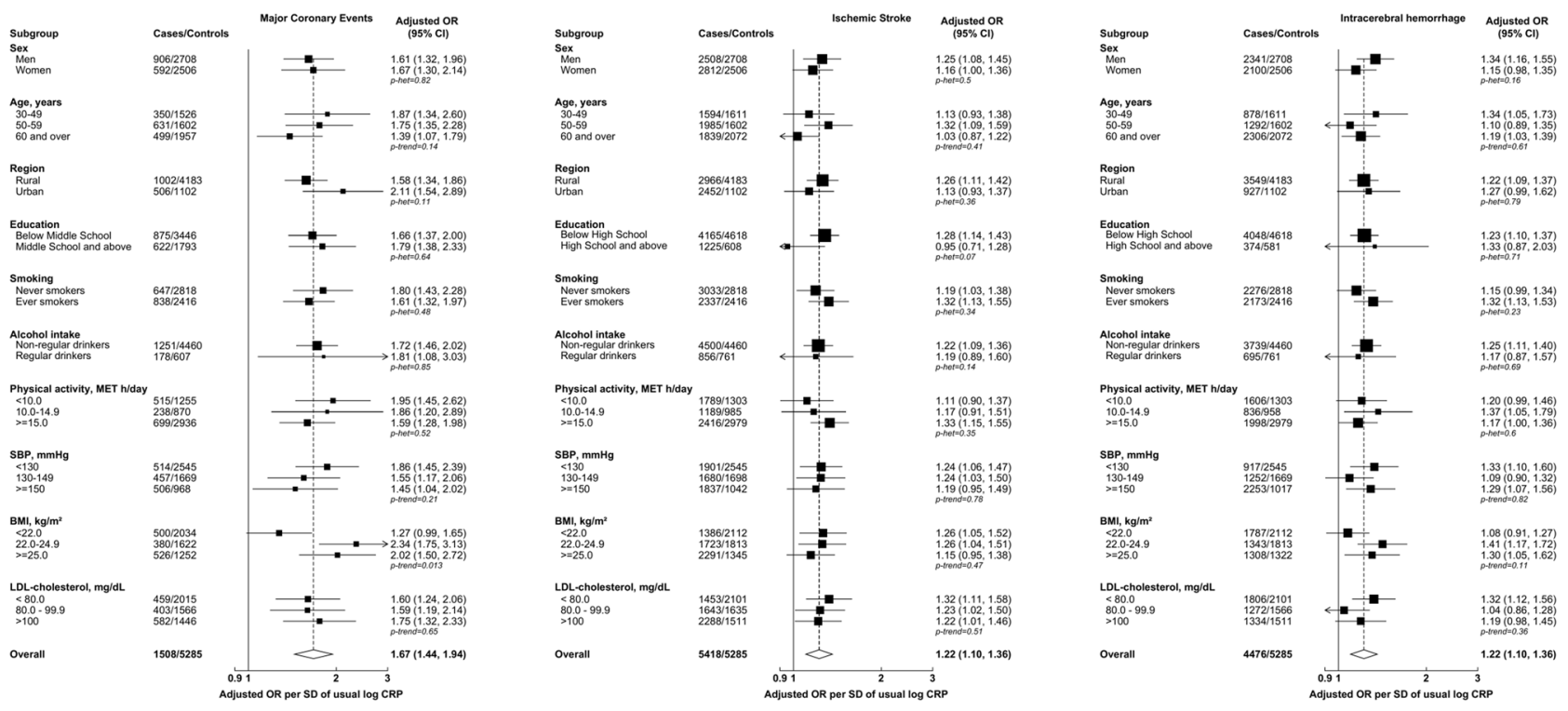

Figure 4. Risk of Major Coronary Events, Ischemic Stroke, and Intracerebral hemorrhage per standard deviation (SD) of usual log-transformed high-sensitivity C-reactive protein (CRP) by pre-specified subgroups. Models were adjusted (where appropriate) for age, age ${ }^{2}$ and sex (base model) and additionally for income, occupation, education, SBP, BMI, diabetes, physical activity, standing height, smoking, alcohol, LDL-C and TG. The models were fitted separately for each of the 10 study areas in each subgroup strata and estimates were meta-analyzed; in each subgroup strata, study areas were excluded when models failed to converge (62 models out of 750 models). Error bars represent $95 \%$ confidence intervals (CI). The sizes of the boxes are proportional to the inverse of the variance of the log ORs. The dotted line represents the overall adjusted OR. The open diamond represents the overall adjusted OR and its 95\% CI. MET: metabolic equivalent of task; $p$-het: $p$-value for heterogeneity; $p$-trend: $p$-value for trend.

and ICH likely reflect roles for biomarkers upstream in the inflammatory signaling pathway (e.g. IL-6 or IL-1 $\beta$ ), as has been shown previously for CHD and $\mathrm{IS}^{17,41,42}$.

\section{Methods}

Study population. China Kadoorie Biobank is a prospective study of 512,715 adults (aged 30-79 years at enrolment). CKB survey methods and the cohort have been described in detail previously ${ }^{29,43}$. The baseline survey recruited participants between 25 June 2004 and 15 July 2008 from 10 diverse regions ( 5 urban and 5 rural) of China. Ethical approval for CKB was obtained from the University of Oxford, the Chinese Centre for Disease Control and Prevention (CCDC) and the regional CDC from the 10 study areas and was conducted according to the Declaration of Helsinki. Ethical approval for CKB study was obtained from the University of Oxford (Oxford Tropical Research Ethics Committee), the Chinese Centre for Disease Control and Prevention (CCDC) and the regional CDC from the 10 study areas. All eligible participants provided informed written consent for participation in the CKB study. Release 15 of the CKB database was used for all analyses.

Data collection. Following informed written consent, study participants (at baseline and resurveys of approximately 5\%) were interviewed on socio-demographics (e.g. age, occupation), lifestyle (e.g. smoking, alcohol consumption) and past medical history (e.g. diabetes, hypertension). Anthropometric (e.g. height, weight, hip and waist circumferences) and physiological measurements (e.g. blood pressure) were obtained using calibrated instruments and following a standard protocol. A $10 \mathrm{~mL}$ non-fasting blood sample (with last meal time recorded) was collected from all participants at baseline and a subset of study participants during resurveys and stored on ice until processing and cryopreservation.

Follow up for fatal and non-fatal outcome. Participants were followed up for incident events, using their unique national identification numbers, by electronic linkage to mortality and disease (CHD, stroke, cancer, and diabetes) registries and to a nationwide health insurance system. These events were coded using the International Classification of Diseases and Related Health Problems, Tenth Revision (ICD-10). Medical records for hospitalization due to CVD events have been systematically reviewed by clinicians in Oxford and China; approximately $90 \%$ of stroke diagnoses were supported by computed tomography (CT) scan or magnetic resonance imagining $(\mathrm{MRI})^{40}$.

Selection of cases and controls. Study participants included in this study were a subset of a larger nested case-control study of incident stroke and ischemic heart disease (IHD) for which participants were selected in June 2013, comprising those for whom clinical chemistry measurements were available from their baseline 
plasma samples; individuals with a history of CVD or cancer at baseline were excluded from both cases and controls. One individual with missing data on BMI was also excluded from all analyses (Fig. S1).

Cases were those whose first CVD incident event was a fatal or nonfatal non-traumatic ICH (ICD-10 codes: I61, I69.1), IS (ICD-10 codes: I63, I69.3), or MCE (defined as acute myocardial infarction [ICD-10: I21-I23] or fatal IHD [ICD-10: I20, I24 or I25] or undergoing a coronary revascularization procedure such as stent placement or coronary artery bypass graft) before $1^{\text {st }}$ January 2017. Those who had two or more of MCE, IS and/or ICH events on the same day, or who had had earlier CVD events other than MCE, IS or ICH during follow-up, were excluded. Common controls were those with no CVD event (endpoint definitions are provided in Table S1) before $1^{\text {st }}$ January 2017.

Measurement of biomarkers. Clinical biochemistry, including markers of inflammation (high-sensitivity CRP [hsCRP], and fibrinogen), lipids (total cholesterol, LDL-C, triglycerides [TG], high-density lipoprotein cholesterol [HDL-C]), lipoproteins (lipoprotein(a) [Lp(a)], apolipoprotein A1 [ApoA1] and apolipoprotein B [ApoB]), markers of liver function (albumin, aspartate transaminase [AST], alanine transaminase [ALT] and gamma-glutamyl transferase [GGT]), markers of renal function (creatinine, uric acid and cystatin-C), and total vitamin D, were assayed at the NDPH Wolfson Laboratory, Oxford. Assays were performed using an AU680 clinical chemistry analyser (Beckman-Coulter, UK) with the following exceptions: plasma fibrinogen and cystatin C were measured using a BN Prospec nephelometer analyser (Siemens, UK); and vitamin D was measured using an Access 2 Immunoanalyser (Beckman-Coulter, UK). All assays used standard manufacturers' reagents, calibrators, and settings.

Amongst study participants, baseline hsCRP (fibrinogen) measurements were available for $1,508(1,280)$ participants with MCE as the first CVD event, 5,418 (2,280) participants with IS as the first CVD event, 4,476 $(2,011)$ participants with ICH as the first CVD event, and 5,285 (3,028) participants with no CVD event before $1^{\text {st }}$ January 2017 (Fig. S1). Repeat measurements of hsCRP and fibrinogen were obtained in a randomly selected subset of individuals using plasma samples collected during the second resurvey in 2013-2014 (hsCRP, $n=1,296$; fibrinogen, $n=639$ ).

Statistical analysis. For all analyses, data for hsCRP, fibrinogen, and selected biomarkers (TG, AST, ALT, GGT, Lp(a), creatinine, cystatin-C) were log-transformed. Adjusted means and proportions of baseline characteristics for cases and controls, and cross-sectional mean levels of hsCRP and fibrinogen for categories (or quintiles) of selected CVD risk factors, were standardized (as appropriate) to age, sex, study area, and case status using $\mathrm{R}$ package lsmeans ${ }^{44}$ with $P$ values for association derived from linear regression models (Wald test). To assess cross-sectional associations of quantitative traits (standardized using the R scale function) per standard deviation (SD) of log-transformed hsCRP and fibrinogen, we used linear regression, adjusted for age and sex, and stratified by study area, combining the area-specific estimates with inverse variance-weighted fixed-effect meta-analysis using function $r m a$ from R package metafor ${ }^{45}$.

To estimate odds ratios (ORs) for the associations of hsCRP and fibrinogen with risk of incident MCE, IS and $\mathrm{ICH}$, logistic regression models were used with adjustment for age, age ${ }^{2}$, and sex (base model), and stratification by study area with inverse variance-weighted fixed effect meta-analysis. The base model was further adjusted (individually or in combination) for body-mass index (BMI), systolic blood pressure (SBP), LDL-C, TG, and other CVD risk factors: household income (in yuan: <2500, 2500-4999, 5000-9999, 10,000-19,999, 20,000$34,999,>35,000$ ); occupation (agriculture, factory worker, administrator/manager, professional/technical, sales and service, retired, house-wife/husband, self-employed, unemployed, other or not stated); education (no formal school, primary school, middle school, high school, technical school/college, university); standing height (meters); self-reported or screen-detected diabetes status ${ }^{46}$; physical activity (metabolic equivalent of task hours per day, MET h/day) ${ }^{47}$; smoking (never smoker, occasional smoker, ex-regular smoker, smoker); and alcohol consumption (never regular drinker, ex-regular drinker, occasional or seasonal drinker, monthly drinker, reduced intake, weekly drinker $)^{48}$. Where models failed to converge due to very low numbers of cases, those study area(s) or strata were excluded from the analysis. The Wald $\chi^{2}$ statistic (with one degree of freedom) was calculated using function anova.rma from $\mathrm{R}$ package metafor to assess the improvement in model fit following inclusion of additional covariates. Heterogeneity and trend tests were conducted by $\chi^{2}$ tests applied to the log ORs and their SEs. For quintiles of hsCRP and fibrinogen, floating CIs were estimated using the Plummer method ${ }^{49}$ to provide $95 \%$ $\mathrm{CI}$ for all quintiles including the reference quintile, enabling inter-quintile comparisons.

For tests of the potential discriminatory utility of hsCRP for MCE, IS, and ICH, C-statistic using the area under the receiver-operating characteristic curve (AUC) was calculated and DeLong's test was applied, using the roc and roc.test functions, respectively, from $\mathrm{R}$ package pROC. Likelihood ratio tests were performed using the $\mathrm{R}$ anova function. Net Reclassification Improvements were estimated using the reclassification function from $\mathrm{R}$ package PredictABEL.

Regression calibration was used to adjust for within-person variation in hsCRP and fibrinogen. Regression coefficients and predicted usual values were estimated from linear regression of log-transformed hsCRP or fibrinogen levels from resurvey samples (taken median 5 years after baseline) on the corresponding baseline values for the same participants. Quintile plots used predicted usual values for CRP and fibrinogen levels; adjusted log ORs per SD of usual log CRP and fibrinogen levels and corresponding standard errors were calculated by dividing those derived using baseline CRP and fibrinogen levels by the corresponding regression coefficient ${ }^{50}$.

Where stated, significance thresholds for $P$ values from multiple hypothesis testing were corrected using the Bonferroni method. Analyses were performed using R version 3.5.0.

\section{Ethical approval and informed consent.}

1. Approval: Ethical approval for $\mathrm{CKB}$ was obtained from the University of Oxford, the Chinese Centre for Disease Control and Prevention (CCDC) and the regional CDC from the 10 study areas 
2. Accordance: Study conducted according to the Declaration of Helsinki.

3. Informed consent: All study participants provided written informed consent for participation in CKB

\section{Data availability}

CKB data, including data used for this study, are available on application according to the CKB data access policy. Data access is overseen by an independent data access committee. Further details can be found here: https://www. ckbiobank.org/site/Data+Access

Received: 1 September 2019; Accepted: 12 March 2020;

Published online: 27 March 2020

\section{References}

1. Roth, G. A. et al. Global, Regional, and National Burden of Cardiovascular Diseases for 10 Causes, 1990 to 2015. J. Am. Coll. Cardiol. 70, 1-25 (2017)

2. Stewart, J., Manmathan, G. \& Wilkinson, P. Primary prevention of cardiovascular disease: A review of contemporary guidance and literature. JRSM Cardiovasc. Dis. 6, 2048004016687211 (2017).

3. Ridker, P. M. Clinician's Guide to Reducing Inflammation to Reduce Atherothrombotic Risk: JACC Review Topic of the Week. J. Am. Coll. Cardiol. https://doi.org/10.1016/j.jacc.2018.06.082 (2018).

4. Tousoulis, D., Charakida, M. \& Stefanadis, C. Endothelial function and inflammation in coronary artery disease. Heart 92, 441-444 (2006).

5. Ruparelia, N., Chai, J. T., Fisher, E. A. \& Choudhury, R. P. Inflammatory processes in cardiovascular disease: a route to targeted therapies. Nat. Rev. Cardiol. 14, 133-144 (2017).

6. Swirski, F. K. \& Nahrendorf, M. Leukocyte behavior in atherosclerosis, myocardial infarction, and heart failure. Science 339, 161-166 (2013).

7. Ramji, D. P. \& Davies, T. S. Cytokines in atherosclerosis: Key players in all stages of disease and promising therapeutic targets. Cytokine Growth Factor. Rev. 26, 673-685 (2015).

8. Willerson, J. T. \& Ridker, P. M. Inflammation as a cardiovascular risk factor. Circulation 109, II2-10 (2004)

9. Ridker, P. M. et al. Antiinflammatory Therapy with Canakinumab for Atherosclerotic Disease. N. Engl. J. Med. 377, 1119-1131 (2017).

10. Ridker, P. M. Targeting inflammatory pathways for the treatment of cardiovascular disease. Eur. Hear. J. 35, 540-543 (2014).

11. Agrawal, A., Hammond, D. J. Jr. \& Singh, S. K. Atherosclerosis-related functions of C-reactive protein. Cardiovasc. Hematol. Disord. Drug. Targets 10, 235-240 (2010).

12. Abd, T. T. et al. The role of C-reactive protein as a risk predictor of coronary atherosclerosis: implications from the JUPITER trial. Curr. Atheroscler. Rep. 13, 154-161 (2011).

13. Blake, G. J. \& Ridker, P. M. C-reactive protein, subclinical atherosclerosis, risk cardiovascular events. Arter. Thromb. Vasc. Biol. 22, $1512-1513$ (2002).

14. Quaglia, L. A. et al. C-reactive protein is independently associated with coronary atherosclerosis burden among octogenarians. Aging Clin. Exp. Res. 26, 19-23 (2014).

15. Zhang, Y. et al. Higher fibrinogen level is independently linked with the presence and severity of new-onset coronary atherosclerosis among Han Chinese population. PLoS One 9, e113460 (2014).

16. Green, D., Foiles, N., Chan, C., Schreiner, P. J. \& Liu, K. Elevated fibrinogen levels and subsequent subclinical atherosclerosis: the CARDIA Study. Atherosclerosis 202, 623-631 (2009).

17. Emerging Risk Factors Collaboration. et al. C-reactive protein concentration and risk of coronary heart disease, stroke, and mortality: an individual participant meta-analysis. Lancet 375, 132-140 (2010).

18. Fibrinogen Studies Collaboration. et al. Plasma fibrinogen level and the risk of major cardiovascular diseases and nonvascular mortality: an individual participant meta-analysis. JAMA 294, 1799-1809 (2005).

19. Liu, Y. et al. Relationship between C-reactive protein and stroke: a large prospective community based study. PLoS One 9, e107017 (2014).

20. Saito, I., Maruyama, K. \& Eguchi, E. C-reactive protein and cardiovascular disease in East asians: a systematic review. Clin. Med. Insights Cardiol. 8, 35-42 (2014).

21. Wakugawa, Y. et al. C-reactive protein and risk of first-ever ischemic and hemorrhagic stroke in a general Japanese population: the Hisayama Study. Stroke 37, 27-32 (2006)

22. C. Reactive Protein Coronary Heart Disease Genetics Collaboration. et al. Association between C reactive protein and coronary heart disease: mendelian randomisation analysis based on individual participant data. BMJ 342, d548 (2011).

23. Elliott, P. et al. Genetic Loci associated with C-reactive protein levels and risk of coronary heart disease. JAMA 302, 37-48 (2009)

24. Zacho, J. et al. Genetically elevated C-reactive protein and ischemic vascular disease. N. Engl. J. Med. 359, 1897-1908 (2008).

25. Keavney, B. et al. Fibrinogen and coronary heart disease: test of causality by 'Mendelian randomization'. Int. J. Epidemiol. 35, 935-943 (2006).

26. Sabater-Lleal, M. et al. Multiethnic meta-analysis of genome-wide association studies in $>100000$ subjects identifies 23 fibrinogenassociated Loci but no strong evidence of a causal association between circulating fibrinogen and cardiovascular disease. Circulation 128, 1310-1324 (2013).

27. Ridker, P. M. et al. Effects of Interleukin-1 $\beta$ Inhibition With Canakinumab on Hemoglobin Alc, Lipids, C-Reactive Protein, Interleukin-6, and Fibrinogen. Circulation 126, 2739-2748 (2012).

28. Wang, W. et al. Prevalence, Incidence, and Mortality of Stroke in China: Results from a Nationwide Population-Based Survey of 480 687 Adults. Circulation 135, 759-771 (2017).

29. Chen, Z. et al. China Kadoorie Biobank of 0.5 million people: survey methods, baseline characteristics and long-term follow-up. Int. J. Epidemiol. 40, 1652-1666 (2011).

30. Vickers, A. J., Cronin, A. M. \& Begg, C. B. One statistical test is sufficient for assessing new predictive markers. BMC Med. Res. Methodol. 11, 13 (2011).

31. Danesh, J. et al. C-Reactive Protein and Other Circulating Markers of Inflammation in the Prediction of Coronary Heart Disease. N. Engl. J. Med. 350, 1387-1397 (2004)

32. Bos, M. J. et al. High serum C-reactive protein level is not an independent predictor for stroke: The Rotterdam Study. Circulation 114, 1591-1598 (2006)

33. Tsai, C. F., Anderson, N., Thomas, B. \& Sudlow, C. L. Comparing Risk Factor Profiles between Intracerebral Hemorrhage and Ischemic Stroke in Chinese and White Populations: Systematic Review and Meta-Analysis. PLoS One 11, e0151743 (2016).

34. Wang, X., Dong, Y., Qi, X., Huang, C. \& Hou, L. Cholesterol levels and risk of hemorrhagic stroke: a systematic review and metaanalysis. Stroke 44, 1833-1839 (2013). 
35. Chen, Z. et al. Adiposity and risk of ischaemic and haemorrhagic stroke in 0.5 million Chinese men and women: a prospective cohort study. Lancet Glob. Heal. 6, e630-e640 (2018).

36. Gruppen, E. G. et al. GlycA, a Pro-Inflammatory Glycoprotein Biomarker, and Incident Cardiovascular Disease: Relationship with C-Reactive Protein and Renal Function. PLoS One 10, e0139057 (2015).

37. Holmes, M. V. et al. Lipids, Lipoproteins, and Metabolites and Risk of Myocardial Infarction and Stroke. J. Am. Coll. Cardiol. 71, 620-632 (2018)

38. Everett, B. M. et al. Rationale and design of the Cardiovascular Inflammation Reduction. Trial: a test. Inflamm. hypothesis atherothrombosis. Am. Hear. J. 166, 199-207 e15 (2013).

39. Ridker, P. M. From C-Reactive Protein to Interleukin-6 to Interleukin-1: Moving Upstream To Identify Novel Targets for Atheroprotection. Circ. Res. 118, 145-156 (2016).

40. Lacey, B. et al. Age-specific association between blood pressure and vascular and non-vascular chronic diseases in 0.5 million adults in China: a prospective cohort study. Lancet Glob. Heal. 6, e641-e649 (2018).

41. IL6R. Genetics Consortium Emerging Risk Factors Collaboration et al. Interleukin-6 receptor pathways in coronary heart disease: a collaborative meta-analysis of 82 studies. Lancet 379, 1205-1213 (2012).

42. Interleukin-6 Receptor Mendelian Randomisation Analysis Consortium. et al. The interleukin-6 receptor as a target for prevention of coronary heart disease: a mendelian randomisation analysis. Lancet 379, 1214-1224 (2012).

43. Chen, Z. et al. Cohort profile: the Kadoorie Study of Chronic Disease in China (KSCDC). Int. J. Epidemiol. 34, 1243-1249 (2005).

44. Lenth, R. V. Least-Squares Means: R. Package lsmeans. 2016(69), 33 (2016).

45. Viechtbauer, W. Conducting Meta-Analyses R. Metaf. Package. 2010(36), 48 (2010).

46. Bragg, F. et al. Association of Random Plasma Glucose Levels With the Risk for Cardiovascular Disease Among Chinese Adults Without Known Diabetes. JAMA Cardiol. 1, 813-823 (2016).

47. Bennett, D. A. et al. Association of Physical Activity With Risk of Major Cardiovascular Diseases in Chinese Men and Women. JAMA Cardiol. 2, 1349-1358 (2017).

48. Millwood, I. Y. et al. Alcohol consumption in 0.5 million people from 10 diverse regions of China: prevalence, patterns and sociodemographic and health-related correlates. Int. J. Epidemiol. 42, 816-827 (2013).

49. Plummer, M. Improved estimates of floating absolute risk. Stat. Med. 23, 93-104 (2004).

50. Rosner, B., Willett, W. C. \& Spiegelman, D. Correction of logistic regression relative risk estimates and confidence intervals for systematic within-person measurement error. Stat. Med. 8, 1051-1053 (1989).

\section{Acknowledgements}

The chief acknowledgement is to the collaborators and participants of China Kadoorie Biobank, to the project staff at Beijing, Oxford, and the 10 regional CKB centers, and to the China National Centre for Disease Control and Prevention (CDC) and its regional offices for assisting with the fieldwork. The baseline survey was supported by the Kadoorie Charitable Foundation, Hong Kong. Long-term follow-up and the second resurvey were supported by grants 088158/Z/09/Z and 104085/Z/14/Z from the UK Wellcome Trust, grant 2011BAI09B01 (2012-2014) from the Chinese Ministry of Science and Technology, and grant 81390541 from the Chinese National Natural Science Foundation. The British Heart Foundation, UK Medical Research Council, and Cancer Research UK provided core funding to the Clinical Trial Service Unit and Epidemiological Studies Unit, University of Oxford. Dr Karim is supported by the Nuffield Department of Population Health (NDPH) Studentship for his DPhil. Dr Holmes is supported by a British Heart Foundation Intermediate Clinical Research Fellowship (FS/18/23/33512) and the National Institute for Health Research Oxford Biomedical Research Centre.

\section{Author contributions}

M.V.H., R.G.W. and M.A.K. conceived the study. M.A.K. performed all statistical analyses, under the supervision of R.G.W. and M.V.H. and wrote the first draft of the manuscript. The other co-authors (C.K., D.A.B., I.Y.M., M.R.H., D.A., Z.B., H.D., Y.G., Y.Q., C.Q., I.T., D.S.V., C.W., C.Y., J.L., J.C., R.C., L.L., Z.M.C. and authors part of the China Kadoorie Biobank Collaborative Group) contributed to the data acquisition and interpretation and provided critical feedback on drafts of the manuscript. All authors read and approved the final version of the manuscript.

\section{Competing interests}

The authors declare no competing interests.

\section{Additional information}

Supplementary information is available for this paper at https://doi.org/10.1038/s41598-020-62391-3.

Correspondence and requests for materials should be addressed to M.V.H. or R.G.W.

Reprints and permissions information is available at www.nature.com/reprints.

Publisher's note Springer Nature remains neutral with regard to jurisdictional claims in published maps and institutional affiliations.

(c) (i) Open Access This article is licensed under a Creative Commons Attribution 4.0 International License, which permits use, sharing, adaptation, distribution and reproduction in any medium or format, as long as you give appropriate credit to the original author(s) and the source, provide a link to the Creative Commons license, and indicate if changes were made. The images or other third party material in this article are included in the article's Creative Commons license, unless indicated otherwise in a credit line to the material. If material is not included in the article's Creative Commons license and your intended use is not permitted by statutory regulation or exceeds the permitted use, you will need to obtain permission directly from the copyright holder. To view a copy of this license, visit http://creativecommons.org/licenses/by/4.0/.

(c) The Author(s) 2020 


\section{Consortia}

\section{China Kadoorie Biobank Collaborative Group}

Rory Collins ${ }^{1}$, Depei Liu ${ }^{3}$, Richard Peto ${ }^{1}$, Ruth Boxall ${ }^{1}$, Yumei Chang ${ }^{1}$, Yiping Chen ${ }^{1}$, Simon Gilbert $^{1}$, Alex Hacker ${ }^{1}$, Andri lona ${ }^{1}$, Rene Kerosi ${ }^{1}$, Ling Kong ${ }^{1,2}$, Om Kurmi ${ }^{1}$, Garry Lancaster ${ }^{1}$, Sarah Lewington ${ }^{1}$, Kuang Lin ${ }^{1}$, John McDonnell ${ }^{1}$, Qunhua Nie ${ }^{1}$, Paul Ryder ${ }^{1}$, Sam Sansome ${ }^{1}$, Paul Sherliker ${ }^{1}$, Rajani Sohoni ${ }^{1}$, Becky Stevens ${ }^{1}$, Jenny Wang ${ }^{1}$, Lin Wang ${ }^{1}$, Neil Wright ${ }^{1}$, Ling $\mathrm{Yang}^{1}$, Xiaoming Yang ${ }^{1}$, Pang Yao ${ }^{1}$, Xiao $\mathrm{Han}^{3}$, $\mathrm{Can} \mathrm{Hou}^{3}$, Pei Pei ${ }^{3}$, Chao Liu ${ }^{3}$, Zengchang Pang ${ }^{9}$, Ruqin $\mathrm{Gao}^{9}$, Shanpeng $\mathrm{Li}^{9}{ }^{9}$, Shaojie Wang ${ }^{9}$, Yongmei Liu ${ }^{9}$, Ranran Du ${ }^{9}$, Liang Cheng ${ }^{9}$, Xiaocao Tian ${ }^{9}$, Hua Zhang ${ }^{9}$, Yaoming Zhai ${ }^{9}$, Feng Ning ${ }^{9}$, Xiaohui Sun ${ }^{9}$, Feifei Li ${ }^{9}$, Silu Lv ${ }^{10}$, Junzheng Wang ${ }^{10}$, Wei Hou ${ }^{10}$, Mingyuan Zou ${ }^{11}$, Shichun Yan ${ }^{11}$, Xue Zhou ${ }^{11}$, Bo Yu ${ }^{12}$, Yanjie $\mathrm{Li}^{12}$, Qinai Xu ${ }^{12}$, Quan Kang ${ }^{12}$, Ziyan Guo ${ }^{12}$, Dan Wang ${ }^{13}$, Ximin $\mathrm{Hu}^{13}$, Jinyan Chen ${ }^{13}$, Yan Fu ${ }^{13}$, Xiaohuan Wang ${ }^{13}$, Min Weng ${ }^{14}$, Zhendong Guo ${ }^{14}$, Shukuan Wu ${ }^{14}$, Yilei $\mathrm{Li}^{14}$, Huimei Li ${ }^{14}$, Ming $\mathrm{Wu}^{14}$, Yonglin Zhou ${ }^{15}$, Jinyi Zhou ${ }^{15}$, Ran Tao ${ }^{15}$, Jie Yang ${ }^{15}$, Jian Su ${ }^{15}$, Fang liu${ }^{16}$, Jun Zhang ${ }^{16}$, Yihe $\mathrm{Hu}^{16}$, Yan Lu' ${ }^{16}$, Liangcai Ma ${ }^{16}$, Aiyu Tang ${ }^{16}$, Yujie Hua ${ }^{16}$, Jianrong Jin ${ }^{17}$, Jingchao Liu ${ }^{17}$, Zhenzhu Tang ${ }^{18}$, Naying Chen ${ }^{18}$, Ying Huang ${ }^{18}$, Mingqiang $\mathrm{Li}^{19}$, Jinhuai Meng ${ }^{19}$, Rong Pan ${ }^{19}$, Qilian Jiang ${ }^{19}$, Jian Lan ${ }^{19}$, Yun Liu ${ }^{19}$, Liuping Wei ${ }^{19}$, Liyuan Zhou ${ }^{19}$, Ningyu Chen ${ }^{19}$, Ping Wang ${ }^{19}$, Fanwen Meng ${ }^{19}$, Yulu Oin Sisi Wang ${ }^{19}$, Xianping $\mathrm{Wu}^{20}$, Ningmei Zhang ${ }^{20}$, Xiaofang Chen ${ }^{20}$, Weiwei Zhou ${ }^{20}$, Guojin Luo ${ }^{21}$, Jianguo Li ${ }^{21}$, Xiaofang Chen ${ }^{21}$, Xunfu Zhong ${ }^{21}$, Jiaqiu Liu $^{21}$, Oiang Sun ${ }^{21}$, Pengfei Ge ${ }^{22}$, Xiaolan Ren ${ }^{22}$, Caixia Dong ${ }^{22}$, Hui Zhang ${ }^{23}$, Enke Mao ${ }^{23}$, Xiaoping Wang ${ }^{23}$, Tao Wang ${ }^{23}$, Xi Zhang ${ }^{23}$, Ding Zhang Zhou ${ }^{24}$, Gang Zhou ${ }^{24}$, Shixian Feng ${ }^{24}$, Ling Chang ${ }^{24}$, Lei Fan ${ }^{24}$, Yulian Gao ${ }^{25}$, Tianyou $\mathrm{He}^{25}$, Huarong Sun ${ }^{25}$, $\mathrm{Pan} \mathrm{He}^{25}$, Chen $\mathrm{Hu}^{25}$, Xukui Zhang ${ }^{25}$, Huifang Wu ${ }^{25}$, Min Yu ${ }^{26}$, Ruying Hu ${ }^{26}$, Hao Wang ${ }^{26}$, Weiwei Gong ${ }^{26}$, Meng Wang $^{26}$, Kaixu Xie ${ }^{27}$, Lingli Chen ${ }^{27}$, Dongxia Pan ${ }^{27}$, Qijun Gu ${ }^{27}$, Yuelong Huang ${ }^{28}$, Biyun Chen ${ }^{28}$, LiYin ${ }^{28}$, Huilin Liv ${ }^{28}$, Zhongxi Fu ${ }^{28}$, Qiaohua Xu ${ }^{28}$, Xin Xu ${ }^{29}$, Hao Zhang ${ }^{29}$, Huajun Long ${ }^{29}$ \& Libo Zhang ${ }^{29}$

${ }^{9} \mathrm{Centre} \mathrm{for} \mathrm{Disease} \mathrm{Control} \mathrm{and} \mathrm{Prevention,} \mathrm{Qingdao} \mathrm{Province,} \mathrm{Shandong,} \mathrm{China.}{ }^{10}$ Licang Centre for Disease Control and Prevention, Qingdao Province, Shandong, China. ${ }^{11}$ Centre for Disease Control and Prevention, Heilongjiang Province, Harbin, China. ${ }^{12}$ Nangang Centre for Disease Control and Prevention, Heilongjiang Province, Harbin, China. ${ }^{13}$ Centre for Disease Control and Prevention, Hainan Province, Haikou, China. ${ }^{14}$ Meilan Centre for Disease Control and Prevention, Hainan Province, Haikou, China. ${ }^{15}$ Centre for Disease Control and Prevention, Jiangsu Province, Nanjing, China. ${ }^{16}$ Suzhou Centre for Disease Control and Prevention, Jiangsu Province, Suzhou, China. ${ }^{17}$ Wuzhong Centre for Disease Control and Prevention, Jiangsu Province, Suzhou, China. ${ }^{18}$ Centre for Disease Control and Prevention, Guangxi Province, Nanning, China. ${ }^{19}$ Liuzhou Centre for Disease Control and Prevention, Guangxi Province, Liuzhou, China. ${ }^{20}$ Centre for Disease Control and Prevention, Sichuan Province, Chengdu, China. ${ }^{21}$ Pengzhou Centre for Disease Control and Prevention, Sichuan Province, Pengzhou, China. ${ }^{22}$ Centre for Disease Control and Prevention, Gansu Province, Lanzhou, China. ${ }^{23}$ Maiji Centre for Disease Control and Prevention, Gansu Province, Tianshui, China. ${ }^{24}$ Centre for Disease Control and Prevention, Henan Province, Zhengzhou, China. ${ }^{25}$ Huixian Centre for Disease Control and Prevention, Henan Province, Huixian, China. ${ }^{26}$ Centre for Disease Control and Prevention, Zhejiang Province, Hangzhou, China. ${ }^{27}$ Tongxiang Centre for Disease Control and Prevention, Zhejiang Province, Tongxiang, China. ${ }^{28}$ Centre for Disease Control and Prevention, Hunan Province, Changsha, China. ${ }^{29}$ Liuyang Centre for Disease Control and Prevention, Hunan Province, Liuyang, China. 